\title{
Klein Tunneling in Deformed Honeycomb Lattices
}

\author{
Omri Bahat-Treidel, Or Peleg, Mark Grobman, Nadav Shapira, and Mordechai Segev \\ Department of Physics, Technion-Israel Institute of Technology, Technion City, Haifa 32000, Israel \\ T. Pereg-Barnea \\ Department of Physics, California Institute of Technology, \\ 1200 East California Boulevard, MC114-36, Pasadena, California 91125, USA
}

(Received 21 May 2009; revised manuscript received 17 September 2009; published 12 February 2010)

\begin{abstract}
We study the scattering of waves off a potential step in deformed honeycomb lattices. For deformations below a critical value, perfect Klein tunneling is obtained; i.e., a potential step transmits waves at normal incidence with nonresonant unit-transmission probability. Beyond the critical deformation a gap forms in the spectrum, and a potential step perpendicular to the deformation direction reflects all normally incident waves, exhibiting a dramatic transition form unit transmission to total reflection. These phenomena are generic to honeycomb lattices and apply to electromagnetic waves in photonic lattices, quasiparticles in graphene, and cold atoms in optical lattices.
\end{abstract}

DOI: 10.1103/PhysRevLett.104.063901

PACS numbers: 42.25.Fx, 73.23.-b

Scattering of relativistic fermions is fundamentally different from scattering of nonrelativistic fermions, since the former are described by Dirac's equation which is first order in the momentum (rather than the second order Schrödinger's equation). Relativistic fermions scattered off a potential step of height $V_{0}$ at normal incidence exhibit nonzero transmission probability even when the particle's energy, $\mathcal{E}$, is lower than $V_{0}$ [1]. This behavior of relativistic fermions is called Klein tunneling, and stands in sharp contrast to the intuitive result of quantum mechanics (QM) for nonrelativistic particles, where the transmission probability vanishes completely for $\mathcal{E}<V_{0}$. This unique scattering process has never been observed experimentally, since the strong fields required to observe Klein tunneling of elementary particles are not currently available. However, it has been predicted that charge carriers in graphene, that obey the massless Dirac's equation, exhibit similar behavior [2]. More specifically, it has been predicted that charge carriers experience nonresonant unit transmission in monolayer graphene, and total reflection in bilayer graphene [3]. Experiments with bipolar junctions were able to show very high conductance in the presence of a gate voltage, indicating high transmission probability [4-6]. In addition, unusual transmission properties were predicted for honeycomb photonic crystals $[7,8]$.

Here we study the dynamics of waves in a deformed honeycomb photonic lattice, and, in particular, the tunneling process into a refractive index step. We find that up to a critical deformation, waves scattered off a potential step at normal incidence exhibit nonresonant unit transmission, irrespective of the details of the potential. Surprisingly, deformed honeycomb lattices display perfect tunneling like nondeformed honeycomb lattices as long as the deformation is subcritical. At deformations greater than the critical deformation a gap forms, resulting in nonresonant total reflection for waves at normal incidence, provided the potential step is perpendicular to the deformation direction. As such, our findings introduce a new domain of transport in photonic structures and beyond, displaying nonresonant unit transmission and total reflection. We emphasize that other optical systems that exhibit unit transmission are typically resonant, and are characterized by transmission peaks, e.g., Fabry-Perot etalon. Finally, the analysis presented here is carried out for electromagnetic waves, the results are generic to all honeycomb lattices, and apply to quasiparticles in graphene, and cold atoms in optical lattices.

Paraxial propagation of a monochromatic field envelope $\psi$ in a honeycomb refractive index structure is given by [9]

$$
i \frac{\partial \psi}{\partial z}=-\frac{1}{2 k} \nabla_{\perp}^{2} \psi-\frac{k \delta n(x, y)}{n_{0}} \psi \equiv \hat{H} \psi,
$$

where $\delta n$ is the modulation in the refractive index, $k$ is the wave number, $n_{0}$ the background refractive index, and $k \delta n(x, y) / n_{0}$ is the optical potential with an opposite sign; i.e., light is attracted to higher index of refraction. Since $\delta n$ is $z$ independent, Eq. (1) has solutions of the form $\psi(x, y, z)=U(x, y) \exp (i \beta z)$, where $U$ is a solution of $\hat{H} U=\beta U$, and $\beta$ is the propagation constant which is analogous to the energy in QM. The honeycomb lattice is composed of two triangular sublattices, denoted by $A$ and $B$ [Fig. 1(a)], and is conveniently described by tight binding (TB). Writing the Hamiltonian in Wannier basis, we consider two sets of amplitudes, $a_{n}, b_{n}$, associated with the two sites $A$ and $B$ in each unit cell located at $\boldsymbol{n}$. Assuming only nearest neighbors hopping (coupling), the TB Hamiltonian resulting from Eq. (1) reads [10,11]

$$
H_{0}=-\sum_{\boldsymbol{n}} \sum_{j=1,2,3} t_{j}\left(a_{\boldsymbol{n}}^{*} b_{\boldsymbol{n}+\boldsymbol{\delta}_{j}}+b_{\boldsymbol{n}+\boldsymbol{\delta}_{j}}^{*} a_{n}\right),
$$



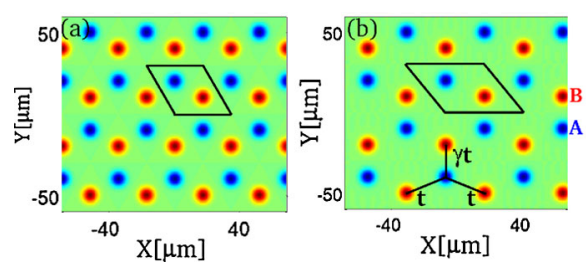

(c)

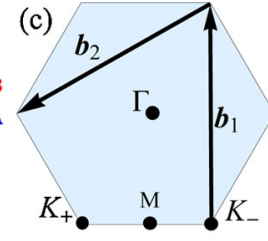

FIG. 1 (color online). Ordinary (a) and deformed (b) honeycomb lattices, with two identical sites in each unit cell. The two sublattices are indicated by $A$ (red or gray) and $B$ (blue or dark gray). (c) The first Brillouin zone, the reciprocal lattice vectors $\vec{b}_{1}, \vec{b}_{2}$, and the Dirac points of nondeformed lattice $K_{ \pm}$.

where the $t_{j}$ 's are the hopping parameters, and $\boldsymbol{\delta}_{j}$ are the vectors connecting the nearest neighbors. The anisotropy is manifested by different hopping parameters in different directions: $t_{2}=t_{3}=t$ and $t_{1}=\gamma t$, where $\gamma>1$ [Fig. 1(b)]. Realizations of such deformed lattices can be implemented by various means [12-14]. We refer to the $y$ direction as the deformation direction since the hopping parameter $t_{1}$ is the greatest [Fig. 1(b)].

Expanding $a_{n}, b_{n}$ in Fourier space and defining $\varphi_{k} \equiv$ $\sum_{j} \exp \left(i \boldsymbol{\delta}_{j} \boldsymbol{k}\right)$, we write

$$
H_{0}=\frac{1}{N} \sum_{\mathrm{BZ}} \Psi^{\dagger} \mathcal{H}_{k} \Psi, \quad \mathcal{H}_{k} \equiv \sigma_{x} \operatorname{Re}\left\{\varphi_{k}\right\}+\sigma_{y} \operatorname{Im}\left\{\varphi_{k}\right\},
$$

where $\sigma_{x}, \sigma_{y}$ are Pauli matrices and $\Psi^{\dagger}=\left(a_{k}^{*} b_{k}^{*}\right)$ is a pseudospinor. The spectrum of propagation constants (energies) is given by the eigenvalues of $\mathcal{H}_{k}$ yielding positive and negative branches:

$$
\beta= \pm t \sqrt{2+\gamma^{2}+4 \gamma \cos \frac{k_{x} a}{2} \cos \frac{\sqrt{3} k_{y} a}{2}+2 \cos k_{x} a},
$$

where $a$ is the lattice constant. For $\gamma \leq 2$, the two branches intersect at two inequivalent points $K_{ \pm}$in the first Brillouin zone, known as the Dirac points. The vicinity of $K_{ \pm}$are known as "valleys," and are associated with the chirality degree of freedom [10]. Hence, each excitation near $K_{ \pm}$is characterized by 4 degrees of freedom: two are associated with the bands and two are associated with the valleys. As $\gamma$ increases, the Dirac points move towards each other until they merge at the critical value: $\gamma_{c}=2$. The merging occurs at the $M$ point [Fig. 1(c)], and for $\gamma>2$ a gap forms [12-15]. An effective Hamiltonian is obtained by expanding $\varphi_{k}$ around $K_{ \pm}$, and its form strongly depends on the deformation strength. For $1 \leq \gamma<2$, the effective Hamiltonian is an anisotropic Dirac's Hamiltonian [12]

$$
\mathcal{H}_{1}=v_{x} p_{x} \sigma_{x}+v_{y} p_{y} \sigma_{y},
$$

where $v_{y}=\sqrt{3} \gamma t a / 2, v_{x}=t a \sqrt{1-\gamma^{2} / 4}$, and $\boldsymbol{p}$ is the momentum measured from the extrema of the bands. As $\gamma$ approaches 2, $v_{x}$ vanishes and one must include high order terms in $p_{x}$. Therefore, at the critical deformation $(\gamma=2)$, the Hamiltonian has no linear term in $p_{x}[16]$

$$
\mathcal{H}_{2}=-\sqrt{3} \operatorname{tap}_{y} \sigma_{y}+\frac{1}{4} t a^{2} p_{x}^{2} \sigma_{x} .
$$

Deriving the effective Hamiltonian for stronger deformations characterized by $\gamma>2$, we find

$$
\begin{aligned}
\mathcal{H}_{3}= & {\left[\Delta+\frac{t a^{2}}{4} p_{x}^{2}-\frac{(8 \gamma-1) t a^{2}}{12} p_{y}^{2}\right] \sigma_{x} } \\
& -\frac{(2 \gamma-1) t a}{\sqrt{3}} \sigma_{y} p_{y},
\end{aligned}
$$

where $\Delta \equiv t(\gamma-2)$ is the gap in the spectrum. The quadratic term in $p_{y}$ may not be neglected, compared to the linear term, since the dispersion obtained from (7) must coincide with the expansion of (4). Note that when the Dirac points merge, the valley degree of freedom vanishes and the number of degrees of freedom is reduced to two. In all three cases, the effective Hamiltonian has the general form $g(\boldsymbol{p}) \sigma_{x}+h(\boldsymbol{p}) \sigma_{y}$, where $g, h$ are functions of $\boldsymbol{p}$. The spinor part of the eigenstates is $\chi_{ \pm}(\boldsymbol{p})=1 / \sqrt{2}(1 \pm(g-$ $i h) /|\beta|$ ), and " \pm " indicates the sign of the propagation constant. In real space, the eigenstates are $\psi_{p}^{( \pm)}(x, y)=$ $\chi^{( \pm)}(\boldsymbol{p}) e^{ \pm i\left(p_{x} x+p_{y} y\right)}$. Having found the eigenstates of the system, we now study scattering off a potential step in deformed honeycomb lattices.

Scattering.-In order to study the scattering problem, we consider a honeycomb lattice with an additional refractive index step with a corresponding optical potential, $V_{N}$. Equation (1) then transforms: $H \rightarrow H-V_{N}$. Consider the scattering of a wave packet composed of Bloch modes from the second band, i.e., $\langle\hat{H}\rangle=-\beta_{0}$. The wave packet is initially located at the region of higher index, and is traveling towards the interface. The height of the step, $V_{0}$, is greater than $\beta_{0}$, as in the scenario considered by Klein. Consider two cases: (i) the step is in the deformation direction ( $y$ direction), and (ii) the step is perpendicular to the deformation direction ( $x$ direction). In both scenarios, we calculate the transmission probability below and above the critical deformation, and find it to be qualitatively different. Below the critical deformation the dynamics are governed by Eq. (5); hence, there is no qualitative difference between potential steps oriented in different directions, i.e., Klein tunneling occurs for a step in any direction. Close to the critical deformation and beyond it, the scattering properties are more complicated and analyzed separately below.

Step in $y$-Consider an additional optical potential $V_{N}(x, y)=V_{0} \Theta(y)$, where $\Theta$ is the Heaviside function, and a wave packet incident from $y \rightarrow-\infty$. The transmission (reflection) probability, $T(R)$, is given by the ratio of the transmitted (reflected) current and the incident current:

$$
R=\left|\mathcal{A}_{r}\right|^{2}, \quad T=\frac{q_{y} \beta_{0}}{p_{y}\left(V_{0}-\beta_{0}\right)}\left|\mathcal{A}_{t}\right|^{2},
$$

where $\mathcal{A}_{t}\left(\mathcal{A}_{r}\right)$ is the transmission (reflection) amplitude obtained from continuity of $\psi$ at the boundary: 


$$
\chi_{-}\left(p_{x},-p_{y}\right)+\mathcal{A}_{r} \chi_{-}\left(p_{x}, p_{y}\right)=\mathcal{A}_{t} \chi_{+}\left(p_{x}, q_{y}\right),
$$

where $q_{y}=\left[\left(V_{0}-\beta_{0}\right)^{2}+v_{x}^{2} p_{x}^{2}\right]^{1 / 2}$. The sign of the momentum in the spinors is determined by the group velocity, which is opposite to the momentum for states of negative $\beta$. With some algebra, close-form expressions for $\mathcal{A}_{t}$ and $\mathcal{A}_{r}$ are obtained; however, these expressions are extremely long and are not included here. At normal incidence, we find a striking result: the transmission probability is exactly 1 for any deformation, ranging from a nondeformed lattice and up to the critical deformation. This result is nonresonant and cannot arise from interference since the waves are scattered from a single interface. Also, unit transmission occurs irrespective of the optical wavelength or the height of the step. We thus find that Klein tunneling occurs not only in nondeformed honeycomb lattices, but rather in a broader class, including honeycomb lattices with weak and strong deformations. This result is an exceptional case in optics, where other cases of unit transmission are resonant, occurring due to interference, and characterized by fine tuning of wavelength and/or the parameters of the potential.

The unique zero backscattering found in graphene was linked to the $\pi$ Berry phase accumulated by winding around the Dirac point [2]. Surprisingly, our findings do not support this linkage; at the critical deformation $(\gamma=2)$ the Dirac points merge and Berry's phase vanishes [15], yet the transmission probability is 1 .

As $\gamma$ increases, we find that the transmission probability becomes less sensitive to the angle of incidence $\theta$, and at the critical deformation $T$ is almost angle independent [Fig. 2(a)] (although $T=1$ only at normal incidence).

Another counterintuitive result obtained is negative refraction. Although the transverse wave vector is conserved, the transverse current changes sign upon penetrating the potential step. Here we find negative refraction with negligible reflection, since close to the critical deformation $T(\theta) \approx 1$ even for large angles. This unique result is in contrast to other systems where negative refraction is typically accompanied by significant reflection. At deformations above the critical one, $T(\theta)$ is always smaller than 1 , but the angular dependence is still extremely weak for large angular range [Fig. 2(a)].

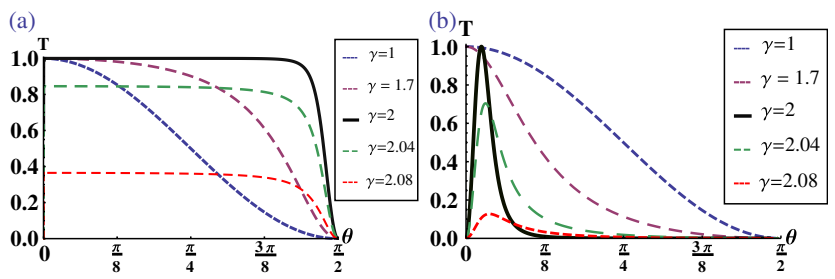

FIG. 2 (color online). Transmission probability as a function of incidence angle, for scattering off a potential step in $y$ (a), and in $x$ (b). The lines correspond to different deformations, above and below the critical value, $\gamma=2$.
Step in $x$-Consider an additional optical potential $V_{N}(x, y)=V_{0} \cdot \Theta(x)$. Well below the critical deformation, the leading term in the Hamiltonian is linear in $p_{x}$, and the behavior is also nonresonant unit transmission at normal incidence. However, as the Dirac points get closer, the Dirac cones are distorted and the valleys are no longer well separated. This situation occurs when the linear term in $p_{x}$ is comparable to the quadratic term. As $\gamma \rightarrow 2$, the quadratic term becomes the leading term and the scattering process changes dramatically: at normal incidence all waves are completely reflected. This dramatic change results from additional exponentially decaying waves. Such waves are present since there are four possible solutions for a specified propagation constant $\beta$ resulting from the quadratic dependence of the Hamiltonian on $p_{x}$ [see Eq. (6)]. The total reflection at normal incidence holds for all deformations beyond the critical one [Fig. 2(b)], even if $V_{0}$ is greater than the gap.

The transition from unit transmission to total reflection is very rapid and occurs in a small range in the parameters space (e.g., deformation strength). This rapid transition might be an indication of a quantum phase transition associated with the merging of the Dirac points [12,15]. To emphasize how rapid the transition is, we note that at $p_{x} a \approx 0.6$ the linear and quadratic terms are equal for $\gamma=$ 1.97, whereas for higher values of $\gamma$ the quadratic term dominates. Therefore, unit transmission occurs for $\gamma \lesssim$ 1.95 , whereas total reflection occurs for $\gamma \geq 2$. The angular dependence is characterized by transmission peaks at small nonzero angles; hence, the potential step filters out the $p_{x}=0$ mode.

The effective Hamiltonian of lattices with deformations close to the critical one resembles the Hamiltonian of bilayer graphene. The scattering properties in the $x$ direction resemble that of bilayer graphene as well [3]. Hence, critically deformed honeycomb lattice exhibits properties of both monolayer and bilayer graphene.

Numerics. - In order to supplement the analytic treatment which includes various assumptions (e.g., tight binding, sharp potential step), we simulate the scattering process, by numerically solving the continuous paraxial wave equation with a honeycomb photonic lattice $[9,13]$ (not relying on tight binding at all). We first solve the eigenvalue problem for the deformed honeycomb lattice (continuous refractive index) [Fig. 1(b)] and find the Bloch waves for deformation close to the critical one. The values of the parameters used in the simulation are $a=35[\mu \mathrm{m}]$, $\max \{\delta n\}=7 \times 10^{-5}, \quad n_{0}=1.5, \quad k=7.85 \times 10^{6}\left[\mathrm{~m}^{-1}\right]$, and the propagation distance $Z=12[\mathrm{~cm}]$. We construct the initial wave packet from Bloch waves of the second band and propagate it by solving Eq. (1) with the additional smooth steplike optical potential. For an index step in the $y$ direction, we find that the entire wave packet is transmitted to the region of lower refractive index, manifesting nonresonant unity transmission in a $2 \mathrm{D}$ system (Fig. 3). 


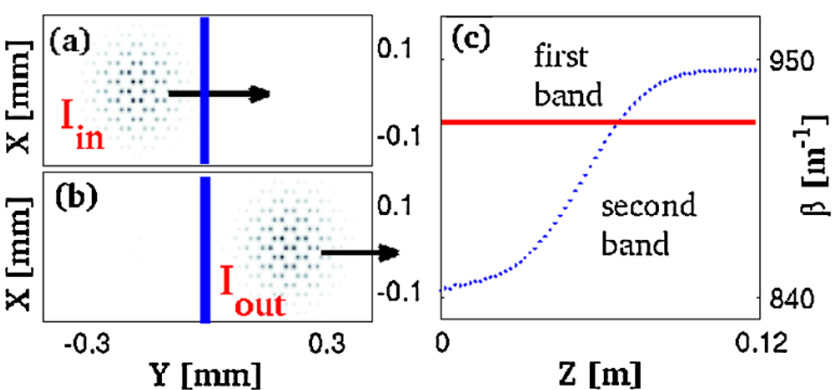

FIG. 3 (color online). The input (a) and output (b) intensities for a step in the $y$ direction, at deformation close to the critical one. The position of the step is indicated by the vertical solid line. (c) The mean propagation constant during the propagation (dots), and the intersection of the bands (solid).

Calculating the mean propagation constant $\langle\hat{H}\rangle$ during propagation verifies that the wave packet transforms from the second to the first band [Fig. 3(c)]. Moreover, we repeat the simulation with various potentials and find that the unit transmission is independent of the shape and width of the potential. As for a step in the $x$ direction, in order to demonstrate total reflection, one must use a very broad beam in the $y$ direction, since waves with even very small momentum in $y$ experience significant transmission. Simulating the scattering process, we find that a wave packet that is completely extended in $y$ (with periodic boundary conditions) undergoes total reflection (Fig. 4). These simulations [(numerically solving Eq. (1)] fully agree with our analytic (tight-binding) findings without any discrepancies.

In conclusion, we found perfect Klein tunneling in deformed honeycomb lattices with subcritical deformations, that is, Klein tunneling occurs in a class of lattices and not solely in nondeformed honeycomb lattice. The scattering process in deformed honeycomb lattices is extremely unique and is divided into two regimes, (i) below the critical deformation; the system exhibits nonresonant unit transmission at normal incidence, for a potential step in any direction; (ii) beyond the critical deformation; all normally incident waves are totally reflected from a potential step perpendicular to the deformation direction. For such a potential step which is perpendicular to the deformation direction, the transmission probability undergoes a very sharp transition from unit transmission (below the critical deformation) to total reflection (beyond the critical deformation).

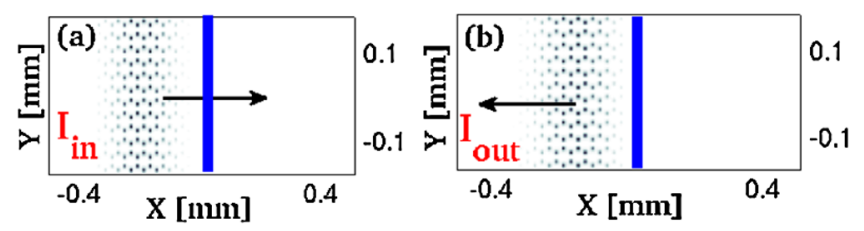

FIG. 4 (color online). The input (a) and output (b) intensities for a step in the $x$ direction. The position of the step is indicated by the vertical solid line.

Finally, our analysis was carried out in the context of photonic lattices where it is possible to observe Klein tunneling directly. However, the results are general and applicable to other fields, such as graphene and cold atoms.

This work was supported by an Advanced Grant from the European Research Council (ERC) and by the Israel Science Foundation (ISF).

[1] O. Klein, Z. Phys. 53, 157 (1929).

[2] T. Ando, T. Nakanishi, and R. Saito, J. Phys. Soc. Jpn. 67, 2857 (1998).

[3] M. I. Katsnelson et al., Nature Phys. 2, 620 (2006).

[4] B. Huard et al., Phys. Rev. Lett. 98, 236803 (2007).

[5] N. Stander, B. Huard, and D. Goldhaber-Gordon, Phys. Rev. Lett. 102, 026807 (2009).

[6] A. F. Young and P. Kim, Nature Phys. 5, 222 (2009).

[7] R. A. Sepkhanov, Y.B. Bazaliy, and C. W. J. Beenakker, Phys. Rev. A 75, 063813 (2007).

[8] R. A. Sepkhanov, J. Nilsson, and C.W.J. Beenakker, Phys. Rev. B 78, 045122 (2008).

[9] O. Peleg, G. Bartal, B. Freedman, O. Manela, M. Segev, and D. N. Christodoulides, Phys. Rev. Lett. 98, 103901 (2007).

[10] V.P. Gusynin, S. G. Sharapov, and J.P. Carbotte, Int. J. Mod. Phys. B 21, 4611 (2007).

[11] M. J. Ablowitz, S. D. Nixon, and Y. Zhu, Phys. Rev. A 79, 053830 (2009).

[12] S.-L. Zhu, B. Wang, and L. M. Duan, Phys. Rev. Lett. 98 , 260402 (2007).

[13] O. Bahat-Treidel, O. Peleg, and M. Segev, Opt. Lett. 33, 2251 (2008).

[14] V. M. Pereira, A. H. Castro Neto, and N. M. R. Peres, Phys. Rev. B 80, 045401 (2009).

[15] B. Wunsch, F. Guinea, and F. Sols, New J. Phys. 10, 103027 (2008).

[16] P. Dietl, F. Piechon, and G. Montambaux, Phys. Rev. Lett. 100, 236405 (2008). 